\title{
Review of: "Microparticles and PD1 interplay added a prognostic impact in treatment outcomes of patients with multiple myeloma"
}

\author{
Diana Castaño ${ }^{1}$ \\ 1 Universidad de Antioquia
}

Potential competing interests: The author(s) declared that no potential competing interests exist.

This article evaluate the expression of PD-1 in extracellular vesicles (EVs) of patients with multiple myeloma. Although the question addressed is very relevant and pertinent in the field, I have different concerns in the isolation and characterization of EVs.

1. During isolation of EVs the authors did not deplete platelets before doing the enrichment of these structures. Therefore, it is very possible that the samples were contaminated with platelets.

2. The authors do not include results about the characterization of the EVs to be sure about their topology (vesicular structure), size, and purity (presence of lipoproteins and plasmatic proteins, analysis of EVs above the electronic noise in the flow cytometer, and the presence of protein aggregates of antibodies or salt crystals in buffers).

3. Following the new guidelines of ISEV, at least two different technics are required for reporting the characterization of extracellular vesicles (DOI: 10.1080/20013078.2018.1535750); the authors only used flow cytometry.

4. The authors do not follow the new nomenclature suggested by ISEV to name the EVs (DOI:

10.1080/20013078.2018.1535750). There is not experiments in the manuscript that demonstrate this EVs are truly microparticles and not platelets or other type of vesicles, such as exosomes, oncosomes and apoptotic bodies.

5. Not all EVs are annexin $\mathrm{V}+$, therefore it is important to include other EV markers to this characterization. In addition, authors are arbitrarily excluding an important proportion of EVs in their analysis without an apparent reason.

In addition, and as a minor points, the authors did not report the company of many reagents and do not include the clones of the antibodies. There is still room to improve the text edition in different parts of the manuscript, including the abstract. 